\title{
LATCH como ferramenta sistematizada para avaliação da técnica de amamentação na maternidade
}

\section{LATCH as a systematic tool for assessment of the breastfeeding technique in maternity LATCH como herramienta sistematizada para evaluar la técnica de lactancia en la maternidad}

Cristiane Maria da Conceição Griffin ${ }^{1}$ ic https://orcid.org/ 0000-0002-5477-0495

Maria Helena Costa Amorim² id hittps://orcid.org/ 0000-0002-4252-7092

Fabiane de Amorim Almeida ${ }^{3}$ id https://orcid.org/ 0000-0002-8062-3579

Karla Oliveira Marcacine ${ }^{2}$ iD https://orcid.org/ 0000-0003-2373-7980

Rosely Erlach Goldman² id https://orcid.org/ 0000-0003-4011-1875

Kelly Pereira Coca² ${ }^{2}$ https://orcid.org/ 0000-0002-3604-852X

Como citar: , Marcacine KO, sist sistematizada para avaliação da técnica de amamentação na maternidade. Acta Paul Enferm. 2022;35:APE03181.

http://dx.doi.org/10.37689/acta-ape/2022A003181

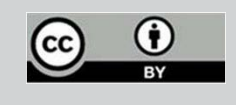

Descritores

Aleitamento materno; Alojamento conjunto; Recém-nascido; Período pós-parto; Avaliação em enfermagem

Keywords

Breast feeding; Rooming-in care; Infant, newborn; Postpartum period; Nursing assessment

Descriptores

Lactancia materna; Alojamiento conjunto; Recién nacido; Periodo pós-parto; Evaluación en enfermería

Submetido 21 de Outubro de 2020

Aceito

21 de Maio de 2021

Autor correspondente Cristiane Maria da Conceição Griffin E-mail: ckristtal@gmail.com

Editor Associado (Avaliação pelos pares): (https://orcid.org/0000-0002-6243-6497) Escola Paulista de Enfermagem, Universidade Federal de São Paulo, São Paulo, SP, Brasil

\section{Resumo}

Objetivo: Analisar as dificuldades das mulheres relacionadas à técnica de amamentação, segundo a escala LATCH e verificar relações com as características sociodemográficas, obstétricas e neonatais.

Métodos: Estudo analítico transversal com mulheres e respectivos filhos únicos em aleitamento materno exclusivo entre junho e dezembro de 2015. Os escores individuais e total da escala LATCH foram usados para avaliar a técnica de amamentação, considerando-se as dificuldades das mulheres para amamentar e as horas de vida da criança. Os testes Qui-quadrado, exato de Fisher, Kruskal-Wallis e um modelo linear generalizado foram usados para avaliar as relações entre os escores da LATCH e as características sociodemográficas, clínicas e obstétricas.

Resultados: Dentre as 162 duplas mãe-filho analisadas, as crianças com mais 48 horas de vida apresentaram menos dificuldades em relação à pega $(p=0,002)$, à deglutição audível $(p<0,001)$ e ao posicionamento $(p<0,001)$. No item conforto, as puérperas com filhos com $<24$ horas de vida apresentaram menos dor $(p=0,004)$. 0 LATCH foi menor para as mulheres com cirurgia mamária prévia $(p=0,005)$, com filhos prematuros $(p=0,011)$, peso menor de 2.500 gramas $(p=0,006)$ e com $<24$ horas de vida.

Conclusão: 0 uso do LATCH foi útil na análise das dificuldades da técnica de amamentação das mulheres durante a fase da internação, considerando as características da mulher e da criança.

\section{Abstract}

Objective: To analyze women's difficulties related to the breastfeeding technique according to the LATCH tool and assess the relationships with sociodemographic, obstetric and neonatal characteristics.

Methods: Cross-sectional analytical study with women in a single birth and their children in exclusive breastfeeding between June and December 2015. The individual and total scores of the LATCH tool were used to assess the breastfeeding technique, considering women's difficulties to breastfeed and the child's hours of life. Chi-square, Fisher's exact, Kruskal-Wallis tests, and a generalized linear model were used to assess the relationships between LATCH scores and sociodemographic, clinical, and obstetric characteristics. Results: Among the 162 mother-child pairs analyzed, children over 48 hours of life presented less difficulties in relation to latching $(p=0.002)$, audible swallowing $(p<0.001)$ and positioning $(p<0.001)$. In the comfort item, women with infants $<24$ hours of life had less painful ( $p=0.004)$. The LATCH score was lower for women with previous breast surgery $(p=0.005)$, with premature children $(p=0.011)$ weighing less than 2,500 grams $(p=0.006)$ and aged $<24$ hours of life.

Conclusion: The LATCH tool was useful in analyzing women's difficulties with the breastfeeding technique during the hospitalization phase, considering the characteristics of the woman and the child. 


\section{Resumen}

Objetivo: Analizar las dificultades de las mujeres relacionadas con la técnica de lactancia, de acuerdo con la escala LATCH y verificar relaciones con características sociodemográficas, obstétricas y neonatales.

Métodos: Estudio analítico transversal con mujeres y sus respectivos hijos únicos en lactancia materna exclusiva entre julio y diciembre de 2015. La puntuación individual y total de la escala LATCH fue utilizada para evaluar la técnica de lactancia, considerando las dificultades de las mujeres para amamantar y las horas de vida del bebé. Para evaluar las relaciones entre la puntuación de LATCH y las características sociodemográficas, clínicas y obstétricas, se utilizó la prueba $\chi^{2}$ de Pearson, la prueba exacta de Fisher, la prueba de Kruskal-Wallis y un modelo lineal generalizado.

Resultados: Entre los 162 binomios madre e hijo analizados, los niños con más de 48 horas de vida presentaron menos dificultades con relación a la prendida $(p=0,002)$, a la deglución audible $(p<0,001)$ y a la colocación $(p<0,001)$. En el área comodidad, las puérperas con hijos de $<24$ horas de vida presentaron menor dolor $(p=0,004)$. La puntuación LATCH fue menor en mujeres con cirugía mamaria previa $(p=0,005)$, con hijos prematuros $(p=0,011)$, peso inferior a 2500 gramos $(p=0,006)$ y con $<24$ horas de vida.

Conclusión: El uso de la escala LATCH fue útil para analizar las dificultades de la técnica de lactancia de mujeres durante la fase de internación, considerando las características de la mujer y del niño.

\section{Introdução}

A utilização de um instrumento de avaliação da amamentaçáo aprimora a prática do profissional de saúde à medida que sistematiza e registra sua atuação, facilita a proposta de condutas individualizadas à mãe e seu filho, e qualifica a comunicação escrita entre os profissionais, $o$ que oferece continuidade para as intervençôes. ${ }^{(1)}$

A ferramenta de avaliação LATCH é uma das mais utilizadas na prática clínica, ${ }^{(1)}$ desenvolvida com a finalidade de auxiliar os profissionais de saúde na avaliação das técnicas de amamentação e identificar situações nas quais é necessária uma intervenção a fim de melhorar o atendimento ao binômio máe-bebê. ${ }^{(2)}$

$\mathrm{O}$ escore LATCH é formatado com sistema de grades semelhante à grade de pontuação do Apgar, cuja simplicidade facilita a documentação e a comunicação sistemática entre os profissionais. ${ }^{(2)} \mathrm{A}$ fácil visualização possibilita ao enfermeiro identificar rapidamente os itens de sua intervenção, contribuindo de maneira significativa para o sucesso na prática do aleitamento materno, além de detectar precocemente as dificuldades relacionadas à amamentação, sendo fator facilitador na tentativa de reduzir as taxas do desmame precoce. ${ }^{(3)}$

$\mathrm{O}$ sistema LATCH atribui uma pontuação numérica $(0,1$ ou 2$)$ a cinco componentes-chave da amamentação para uma pontuação total possível de 10 pontos. Sugere-se que a qualidade da pega do bebê deve ser avaliada duas vezes durante um período de 24 horas, por dois profissionais de saúde, e documentada. ${ }^{(2)}$

A ferramenta mostra-se capaz de prever a duração do aleitamento materno logo nas primeiras 24 horas de vida, uma vez que apresenta uma relação significativa entre um escore mais alto e a exclusividade do aleitamento materno. ${ }^{(3)}$ São vitais as avaliações do aleitamento materno desde a primeira mamada na sala de parto, até o momento da alta. ${ }^{(4)}$ Os baixos escores do LATCH indicam a necessidade de intervenção imediata pelo profissional, apoio e acompanhamento pós-alta. ${ }^{(4)}$ A utilização da escala é essencial, e tem mostrado correlação positiva com a duração do aleitamento materno. ${ }^{(4,5)}$ A escala LATCH mostra um elevado grau de concordância entre os avaliadores e uma correlaçáo significativa entre os escores de avaliação de mães e enfermeiras, sendo, portanto, um elemento confiável e objetivo para a avaliação da técnica da amamentação do ponto de vista clínico. ${ }^{(3,6)}$

A sua utilização permite ampliar a autoconfiança materna em relação à sua capacidade de amamentar e lidar com as necessidades de seu filho, e constitui um indicador de qualidade para as instituiçóes de saúde. ${ }^{(7)}$ No Brasil, esta ferramenta é pouco conhecida e explorada na prática clínica, apesar de ter sido traduzida e validada na língua portuguesa. ${ }^{(10)}$

Desta forma, o presente estudo teve como objetivo analisar as dificuldades das mulheres relacionadas à técnica de amamentação, segundo a escala LATCH e verificar relaçóes com as características sociodemográficas, obstétricas e neonatais.

\section{Métodos}

Estudo analítico transversal com mulheres e respectivos filhos nascidos na maternidade de um hospital privado de nível terciário de São Paulo, Brasil. O 
hospital possui um total de 617 leitos e atende diversas áreas. A maternidade dispóe de 56 leitos de alojamento conjunto e uma taxa média de 4.000 partos/ano. A prática da amamentação no serviço é estimulada logo após o nascimento, e ocorre dentre as primeiras 2 horas no Centro Obstétrico. A mamada no alojamento conjunto é estimulada em livre demanda.

Participaram do estudo mulheres que tiveram filhos no respectivo serviço entre junho e dezembro de 2015. Foram incluídas mulheres com filho único, internados na maternidade, em sistema de alojamento conjunto, e que estavam em aleitamento materno exclusivo. Foram excluídas aquelas cujos filhos foram encaminhados para as unidades de Cuidado Intermediário ou de Terapia Intensiva Neonatal durante algum momento da internação, no momento do estudo.

A coleta de dados foi realizada por duas enfermeiras, por meio de obtenção de dados do prontuário, entrevista e observação da mamada. Para tanto, utilizou-se um instrumento específico contendo as seguintes variáveis: dados sociodemográficos da mulher (idade, escolaridade), obstétricos (paridade, tipo de parto, presença e tipo de cirurgia mamária) e dados da criança (horas de vida, sexo, idade gestacional, Apgar no $1^{\circ}$ e $5^{\circ}$ minutos, peso ao nascer e peso atual).

As dificuldades da técnica de amamentação das mulheres para amamentar foi avaliada utilizando-se a escala LATCH. ${ }^{(10)}$ A ferramenta é usada para auxiliar o profissional enfermeiro na avaliação sistematizada da mamada quanto ao tipo de dificuldade apresentada pela mulher e pela criança. A palavra LATCH é o acrônimo em inglês para as característica avaliadas: "L" (latch - pega) refere-se à qualidade da pega da criança na mama; "A" (audible swallowing - deglutição audível) refere-se à possibilidade de se ouvir a deglutição do bebê enquanto está mamando; "T" (type of nipple - tipo de mamilo) avalia o tipo de mamilo; "C" (comfort - conforto) refere-se à queixa materna quanto a dor mamária e presença de dor e/ou lesão mamilar; "H" (hold - posicionamento) refere-se à mãe precisar ou náo de ajuda para posicionar o bebê. A ferramenta LATCH atribui uma pontuação numérica de 0 a 2 a cada um dos cinco componentes-chave da amamentação, cuja pontuação máxima é de 10 pontos (Quadro 1). ${ }^{(10)}$

Quadro 1. Escala LATCH

\begin{tabular}{|c|c|c|c|c|}
\hline & 0 & 1 & 2 & Escore \\
\hline $\begin{array}{l}\text { L } \\
\text { Pega }\end{array}$ & $\begin{array}{l}\text { Muito sonolento ou } \\
\text { relutante } \\
\text { Não consegue } \\
\text { sustentar a pega ou } \\
\text { sucção }\end{array}$ & \begin{tabular}{|l} 
Tentativas repetidas \\
para sustentar a \\
pega ou sucção \\
Segura o mamilo \\
na boca \\
Estimular para \\
sugar
\end{tabular} & \begin{tabular}{|l} 
Pega (agarra) a \\
mama \\
Língua abaixada \\
Lábios curvados \\
para fora \\
Sucção rítmica
\end{tabular} & \\
\hline $\begin{array}{l}\text { A } \\
\text { Deglutiçãa } \\
\text { audível }\end{array}$ & Nenhuma & $\begin{array}{l}\text { Um pouco, com } \\
\text { estímulo }\end{array}$ & $\begin{array}{l}\text { Espontânea e } \\
\text { intermitente }(<24 \\
\text { horas de vida) } \\
\text { Espontânea e } \\
\text { frequente }(>24 \\
\text { horas de vida) }\end{array}$ & \\
\hline $\begin{array}{l}\text { T } \\
\text { Tipo de mamilo }\end{array}$ & Invertido & $\begin{array}{l}\text { Semiprotruso } \\
\text { (plano) }\end{array}$ & $\begin{array}{l}\text { Protruso (após } \\
\text { estimulação) }\end{array}$ & \\
\hline $\begin{array}{l}\text { C } \\
\text { Conforto } \\
\text { (Mama/mamilo) }\end{array}$ & $\begin{array}{l}\text { Ingurgitada } \\
\text { Com fissura, } \\
\text { sangrando, grandes } \\
\text { vesículas ou } \\
\text { equimoses. } \\
\text { Desconforto severo }\end{array}$ & $\begin{array}{l}\text { Cheia } \\
\text { Avermelhado/ } \\
\text { pequenas vesículas } \\
\text { ou equimoses. } \\
\text { Desconforto suave/ } \\
\text { moderado }\end{array}$ & $\begin{array}{l}\text { Macias } \\
\text { Não dolorosas }\end{array}$ & \\
\hline $\begin{array}{l}\text { H } \\
\text { Posicionamento }\end{array}$ & $\begin{array}{l}\text { Ajuda completa } \\
\text { (Equipe segura } 0 \\
\text { bebê à mama) }\end{array}$ & $\begin{array}{l}\text { Ajuda mínima } \\
\text { (por exemplo, } \\
\text { elevar a cabeça na } \\
\text { cabeceira da cama, } \\
\text { colocar travesseiros } \\
\text { para apoio) } \\
\text { Ensinar a mãe em } \\
\text { uma mama, depois } \\
\text { ela faz no outro } \\
\text { lado } \\
\text { Equipe segura o } \\
\text { bebê, depois a mãe } \\
\text { assume }\end{array}$ & $\begin{array}{l}\text { Sem ajuda da } \\
\text { equipe } \\
\text { Mãe capaz de } \\
\text { posicionar e } \\
\text { segurar o bebê }\end{array}$ & \\
\hline
\end{tabular}

Fonte: Conceição CM, Coca KP, Alves MR, Almeida FA. Validação para língua portuguesa do instrumento de avaliação do aleitamento materno LATCH. $^{(10)}$

A coleta de dados ocorreu diariamente. As enfermeiras verificaram as mulheres que estavam amamentando no alojamento conjunto, identificavam as elegibilidades e realizavam o convite para participação do estudo. Após a assinatura do Termo de Consentimento Livre e Esclarecido (TCLE) pela puérpera, a enfermeira realizava a entrevista, seguida da confirmação dos dados em prontuário e, depois, da observação da técnica da amamentação.

Os dados coletados foram transferidos para as planilhas de Excel. As variáveis categóricas foram descritas por frequência, e as numéricas, apresentadas como média, desvio padrão, valores mínimo e máximo. Consideraram-se como variáveis respostas deste estudo: idade materna (categorizada em: $<39$ anos e $\geq 40$ anos), paridade (primípara ou multípara), tipo de parto (vaginal e cesariana), cirurgia mamária prévia (presença ou ausência), tipo de cirurgia mamária (aditiva, redutora ou retirada de nódulo), 
idade gestacional da criança ( $<37$ ou $\geq 37$ semanas), peso ao nascer $(<2.500$ ou $\geq 2.500$ gramas $)$, Apgar $1^{\circ}$ minuto $(<7$ ou $\geq 7)$ e horas de vida da criança ( $<24$ horas, $24-48$ horas ou $>48$ horas). Os grupos de tempo de vida dos recém-nascidos foram comparados em relaçáo às características maternas e dos recém-nascidos por meio de modelos de análise de variância (ANOVA) ou testes não paramétricos de Kruskal-Wallis e as diferenças localizadas por testes de comparaçôes múltiplas com os valores $\mathrm{p}$ corrigidos pelo método de Bonferroni para variáveis numéricas e testes Qui-quadrado ou exatos de Fisher para variáveis categóricas.

As dificuldades para amamentar foram apresentadas e analisadas de acordo com as horas de vida da criança ( $<24$ horas, 24 a 48 horas e $>48$ horas), em relação aos escores individuais e total da escala LATCH.

A associaçáo entre a idade dos recém-nascidos e os escores (individuais e total) da escala LATCH foram realizadas por meio de testes não paramétricos de Kruskal-Wallis, e as diferenças, localizadas por testes de comparaçóes múltiplas com os valores $\mathrm{p}$ corrigidos pelo método de Bonferroni. Associações entre a idade dos recém-nascidos e escore total da escala LATCH, utilizando diferentes pontos de corte, foram investigadas por meio de testes Quiquadrado ou exatos de Fisher.

Foram ajustados modelos lineares generalizados em abordagem simples para a variável resposta escore total na escala LATCH, com distribuição de probabilidade gama e função de ligação log, e as variáveis explicativas: características sociodemográficas, clínicas e obstétricas. Devido à relação importante do tempo de vida da criança no momento da coleta com o escore total da escala LATCH, foram ajustados modelos similares com as mesmas variáveis explicativas, considerando também o tempo de vida da criança no momento da coleta. Os resultados dos modelos foram apresentados por médias estimadas para o escore total e intervalos de confiança de $95 \% .{ }^{(11)}$

Os dados foram analisados a partir do software SPSS, e foi considerado nível de significância de 5\% e intervalo de confiança de $95 \%$. O número de mamadas a serem avaliadas neste estudo foi estimado com o objetivo principal de obter precisão de $0,1(10 \%) \mathrm{em}$ um intervalo de $95 \%$ de confiança para o coeficiente de correlação intraclasse, que foi utilizado para a avaliação da reprodutibilidade do instrumento. Como não há informação acerca desse coeficiente aplicado a essa escala, antecipamos o valor de 0,6 de modo que, para uma precisão de $10 \%$, o número necessário de mamadas foi de 158, de acordo com a metodologia proposta por Shoukri et al. ${ }^{(12)}$

\section{Resultados}

A amostra do estudo foi composta por 162 mulheres e seus respectivos filhos. A idade média das mulheres estudadas foi de 34 anos (DP = 3,8; mín. 24 e máx. 45), 96\% tinham ensino superior completo, $52 \%$ eram primíparas, $72 \%$ tiveram parto cesariana e $19 \%$ apresentavam história de cirurgia mamária prévia (14\% mamoplastia aditiva, 3\% redutora e $2 \%$ retirada de nódulos). Quanto aos dados do recém-nascido, $46 \%$ estavam com 24 a 48 horas vida, $55 \%$ do sexo masculino, a idade gestacional média identificada foi de 39 semanas ( $\mathrm{DP}=1,2, \mathrm{~min} .34 \mathrm{e}$ máx. 41), 95\% deles apresentaram índice de Apgar $\geq 7$ no primeiro minuto e $100 \% \geq 9$ no quinto minuto, o peso ao nascer médio foi de 3.300 gramas ( $\mathrm{DP}=404$, mín. 2.185, máx. 4.420) e o peso médio no dia da coleta dos dados foi de 3.140 gramas (DP = 408, mín. 2.085, máx. 4.290).

Os grupos de tempo de vida dos recém-nascidos foram comparados em relação às características maternas e dos recém-nascidos. Observaram-se diferenças nas médias de idade gestacional ao nascimento e no peso do dia da coleta, o grupo com menos de 24 horas de vida apresentou médias maiores do que o grupo com mais de 48 horas (Tabela 1).

Quanto aos escores individuais, foram observadas diferenças entre os grupos nos escores de pega $(\mathrm{p}=0,002)$, deglutição audível $(\mathrm{p}<0,001)$, conforto $(\mathrm{p}=0,004)$ e posicionamento $(\mathrm{p}<0,001)$ (Tabela 2$)$.

Em relação à dificuldade na pega, os recém-nascidos com $>48$ horas de vida apresentaram escore mais alto de adequação quando comparado ao grupo com $<24$ horas de vida $(\mathrm{p}=0,002)$. Não foram observadas diferenças na comparaçáo entre os ou- 
Tabela 1. Análise da homogeneidade dos grupos segundo horas de vida da criança e características maternas e da criança

\begin{tabular}{|c|c|c|c|c|}
\hline \multirow[b]{2}{*}{ Características } & \multicolumn{3}{|c|}{ Horas de vida da criança } & \multirow[b]{2}{*}{$p$-value } \\
\hline & $\begin{array}{c}<24 \text { horas } \\
n(\%)\end{array}$ & $\begin{array}{c}24 \text { a } 48 \text { horas } \\
n(\%)\end{array}$ & $\begin{array}{c}>48 \text { horas } \\
\mathrm{n}(\%)\end{array}$ & \\
\hline \multicolumn{5}{|l|}{ Maternas } \\
\hline $\begin{array}{l}\text { Idade materna (anos) } \\
\text { Média (DP) }\end{array}$ & $34,0(3,4)$ & $33,5(3,8)$ & $34,1(4,2)$ & $0,668^{*}$ \\
\hline $\begin{array}{l}\text { Escolaridade - Superior } \\
\text { completo }\end{array}$ & $42(97,7)$ & $69(93,2)$ & $44(97,8)$ & $0,417^{\star \star}$ \\
\hline Paridade primípara & $20(46,5)$ & $40(54,1)$ & $25(55,6)$ & $0,651^{\star \star \star}$ \\
\hline Tipo de parto cesariana & $30(69,8)$ & $52(70,3)$ & $34(75,6)$ & $0,924^{\star \star}$ \\
\hline $\begin{array}{l}\text { Cirurgia mamária prévia } \\
\text { - não }\end{array}$ & $32(74,4)$ & $61(82,4)$ & $38(84,4)$ & $0,439^{\star \star \star}$ \\
\hline \multicolumn{5}{|l|}{ Criança } \\
\hline $\begin{array}{l}\text { IG (semanas) } \\
\text { Média (DP) }\end{array}$ & $39,3(1,0)$ & $38,9(1,1)$ & $38,7(1,3)$ & $0,027^{*}$ \\
\hline Apgar $1^{1} \mathrm{~min} . \geq 7$ & $42(97,7)$ & $69(93,2)$ & $43(95,5)$ & $0,286^{\star *}$ \\
\hline Apgar $5^{\circ} \mathrm{min} . \geq 7$ & $43(100)$ & $74(100)$ & $45(100)$ & $0,483^{\star *}$ \\
\hline $\begin{array}{l}\text { Peso ao nascer } \\
\text { Média (DP) }\end{array}$ & $3257,9(355,5)$ & $3357,8(423,7)$ & $3212,9(406,6)$ & $0,136^{*}$ \\
\hline $\begin{array}{l}\text { Peso atual } \\
\text { Média (DP) }\end{array}$ & $3257,9(355,5)$ & $3228,5(412,4)$ & $2993,9(358,4)$ & $0,002^{*}$ \\
\hline
\end{tabular}

IG - Idade Gestacional; DP - desvio padrão; * Modelo de análise de variância; **Teste Exato de Fischer; ***teste Qui-quadrado

Tabela 2. Escores individuais da escala LATCH segundo as dificuldades das mulheres $(n=162)$ e as horas de vida dos recém-nascidos

\begin{tabular}{|c|c|c|c|c|}
\hline \multirow[b]{2}{*}{ Escores } & \multicolumn{3}{|c|}{ Horas de vida da criança } & \multirow[b]{2}{*}{$p$-value } \\
\hline & $\begin{array}{c}<24 \\
(n=43) \\
n(\%)\end{array}$ & $\begin{array}{c}24-48 \\
(n=74) \\
n(\%)\end{array}$ & $\begin{array}{c}>48 \\
(n=45) \\
n(\%)\end{array}$ & \\
\hline L - Pega & & & & 0,002 \\
\hline 0 & $3(7)$ & $6(8,1)$ & $2(4,4)$ & \\
\hline 1 & $18(41,9)$ & $14(18,9)$ & $4(8,9)$ & \\
\hline 2 & $22(51,2)$ & $54(73)$ & $39(86,7)$ & \\
\hline A - Deglutição audível & & & & $<0,001$ \\
\hline 0 & $43(100)$ & $68(91,9)$ & $32(71,1)$ & \\
\hline 1 & $0(0)$ & $4(5,4)$ & $11(24,4)$ & \\
\hline 2 & $0(0)$ & $2(2,7)$ & $2(4,4)$ & \\
\hline T - Tipo de mamilo & & & & 0,062 \\
\hline 0 & $1(2,3)$ & $0(0)$ & $0(0)$ & \\
\hline 1 & $1(2,3)$ & $0(0)$ & $0(0)$ & \\
\hline 2 & $41(95,3)$ & $74(100)$ & $45(100)$ & \\
\hline C - Conforto(mama/mamilo) & & & & 0,004 \\
\hline 0 & $0(0)$ & $0(0)$ & $1(2,2)$ & \\
\hline 1 & $1(2,3)$ & $14(18,9)$ & $12(26,7)$ & \\
\hline 2 & $42(97,7)$ & $60(81,1)$ & $32(71,1)$ & \\
\hline H - Colo (posicionamento) & & & & $<0,001$ \\
\hline 0 & $12(27,9)$ & $5(6,8)$ & $2(4,4)$ & \\
\hline 1 & $22(51,2)$ & $24(32,4)$ & $15(33,3)$ & \\
\hline 2 & $9(20,9)$ & $45(60,8)$ & $28(62,2)$ & \\
\hline
\end{tabular}

$p$-value - teste de Kruskal-Wallis

tros grupos: $<24$ horas com 24-48 horas de vida $(p=0,070)$ e $24-48$ horas com $>48$ horas $(p=0,336)$.

Para a deglutição audível, também foram observadas diferenças na qual os recém-nascidos com $>48$ horas tiveram escore mais alto de positivida- de, quando comparado aos grupos com $<24$ horas $(\mathrm{p}<0,001)$ e com 24-48 horas $(\mathrm{p}=0,002)$. Não se observou diferença na comparação do grupo de $<24$ horas com o de 24-48 horas ( $\mathrm{p}=0,553)$.

Para o item tipo de mamilo náo foram encontradas diferenças entre os grupos de recém-nascidos estudados $(\mathrm{p}=0,062)$.

Para o item conforto observaram-se diferenças entre os grupos, com escores mais altos para os recém-nascidos com $<24$ horas de vida, ao comparar ao grupo com $>48$ horas $(\mathrm{p}=0,004)$. Não foram observadas diferenças entre os grupos: $<24$ horas com o $24-48$ horas $(\mathrm{p}=0,070)$ e 24-48 horas com o $>48$ horas $(\mathrm{p}=0,454)$.

Para o item posicionamento também foram observadas diferenças entre os grupos quanto ao escore, das quais os recém-nascidos com $<24$ horas de vida tiveram menores escores quando comparados aos com 24-48 horas $(p<0,001)$ e com $>48$ horas $(p<0,001)$. $\mathrm{Na}$ comparação entre os grupos com 24-48 horas e $>48$ horas ( $p>0,999)$ não houve diferença.

$\mathrm{Na}$ comparação entre os grupos em relação ao escore total, foram identificadas diferenças $(p<0,001)$, com escores mais baixos para os recém-nascidos com $<24$ horas comparados aos grupos com 24-48 horas $(\mathrm{p}<0,001)$ e com $>48$ horas $(\mathrm{p}<0,001)$. Não foi observada diferença entre os grupos com 24-48 horas e com $>48$ horas $(\mathrm{p}=0,359)$.

Para os demais escores totais, não foram observadas associaçôes. Os recém-nascidos com $>48$ horas apresentaram maior proporção de mamadas com escores $\geq 6, \geq 7$ e $\geq 8$ (Figura 1 ).

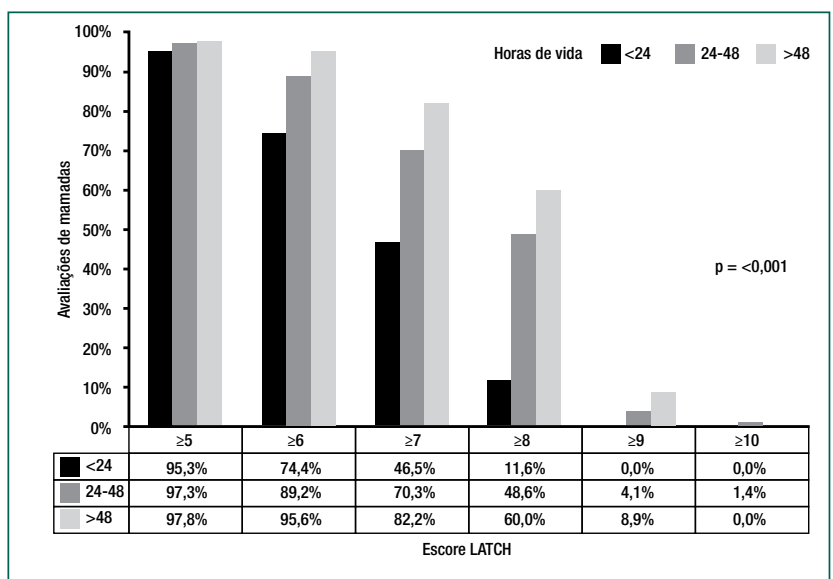

Figura 1. Distribuição do escore total da escala LATCH, segundo 0 tempo de vida dos recém-nascidos no dia da coleta 
No que se refere à distribuição do escore total da escala LATCH, segundo o tempo de vida do recém-nascido para diferentes pontos de corte, foram observadas associaçóes entre o tempo de vida do recém-nascido e o escore total $\geq 6(\mathrm{p}=0,010)$, $\geq 7(\mathrm{p}=0,001)$ e $\geq 8(\mathrm{p}<0,001)$ na escala LATCH (Tabela 3).

Tabela 3. Distribuição dos pontos de corte para o escore total da escala LATCH, segundo o tempo de vida dos recém-nascidos $(\mathrm{n}=162)$

\begin{tabular}{|c|c|c|c|c|}
\hline \multirow[b]{2}{*}{$\begin{array}{l}\text { Escore total } \\
\text { LATCH }\end{array}$} & \multicolumn{2}{|c|}{ Horas de vida } & \multirow[b]{2}{*}{$\begin{array}{c}>48 \\
(n=45) \\
n(\%)\end{array}$} & \multirow[b]{2}{*}{$p$-value } \\
\hline & $\begin{array}{c}<24 \\
(n=43) \\
n(\%)\end{array}$ & $\begin{array}{c}24-48 \\
(n=74) \\
n(\%)\end{array}$ & & \\
\hline & & & & $0,725^{*}$ \\
\hline$<5$ & $2(4,7)$ & $2(2,7)$ & $1(2,2)$ & \\
\hline \multirow[t]{2}{*}{$\geq 5$} & $41(95,3)$ & $72(97,3)$ & $44(97,8)$ & \\
\hline & & & & $0,010^{* *}$ \\
\hline$<6$ & $11(25,6)$ & $8(10,8)$ & $2(4,4)$ & \\
\hline \multirow[t]{2}{*}{$\geq 6$} & $32(74,4)$ & $66(89,2)$ & $43(95,6)$ & \\
\hline & & & & $0,001^{*+}$ \\
\hline$<7$ & $23(53,5)$ & $22(29,7)$ & $8(17,8)$ & \\
\hline \multirow[t]{2}{*}{$\geq 7$} & $20(46,5)$ & $52(70,3)$ & $37(82,2)$ & \\
\hline & & & & $<0,001^{\star}$ \\
\hline$<8$ & $38(88,4)$ & $38(51,4)$ & $18(40,0)$ & \\
\hline \multirow[t]{2}{*}{$\geq 8$} & $5(11,6)$ & $36(48,6)$ & $27(60,0)$ & \\
\hline & & & & $0,128^{*}$ \\
\hline$<9$ & $43(100,0)$ & $71(95,9)$ & $41(91,1)$ & \\
\hline \multirow[t]{2}{*}{$\geq 9$} & $0(0,0)$ & $3(4,1)$ & $4(8,9)$ & \\
\hline & & & & $>0,999^{\circ}$ \\
\hline$<10$ & $43(100,0)$ & $73(98,6)$ & $45(100,0)$ & \\
\hline$\geq 10$ & $0(0,0)$ & $1(1,4)$ & $0(0,0)$ & \\
\hline
\end{tabular}

*Teste exato de Fisher; *^Teste Qui-quadrado

O escore total da escala LATCH foi menor para as mulheres com cirurgia mamária prévia $(\mathrm{p}=0,005)$, cujos filhos apresentavam $<37$ semanas $(\mathrm{p}=0,011)$, peso ao nascer menor de 2.500 gramas $(p=0,006)$ e com $<24$ horas de vida $(\mathrm{p}<0,001)$. O baixo número de mulheres estudadas com cirurgia mamária não permitiu a comparação dessa variável (Tabela 4).

Também se observou uma relação entre a idade da criança e o escore total da escala LATCH. Na idade da criança com 24-48 horas de vida, as mulheres multíparas $(\mathrm{p}=0,032)$, com cirurgia mamária prévia $(\mathrm{p}=0,048)$ e máes de prematuros $(\mathrm{p}=0,006)$ apresentaram menores valores de escore total. Quanto à idade da criança com $>48$ horas de vida, apenas as crianças nascidas prematuras apresentam escores mais baixos $(\mathrm{p}=0,014)$ (Tabela 5).
Tabela 4. Valores médios estimados e intervalos de confiança de $95 \%$ (IC95\%) para o escore total da escala LATCH segundo as variáveis sociodemográficas, clínicas e obstétricas

\begin{tabular}{|c|c|c|}
\hline Variáveis maternas e da criança & Média (IC 95\%) & $p$-value \\
\hline Idade materna & & 0,181 \\
\hline$<40$ anos & $6,9(6,7 ; 7,1)$ & \\
\hline$\geq 40$ anos & $7,6(6,7 ; 8,5)$ & \\
\hline Paridade & & 0,184 \\
\hline Primípara & $7,1(6,8 ; 7,4)$ & \\
\hline Multípara & $6,8(6,6 ; 7,1)$ & \\
\hline Tipo de parto & & 0,136 \\
\hline Vaginal & $7,2(6,8 ; 7,6)$ & \\
\hline Cesárea & $6,9(6,7 ; 7,1)$ & \\
\hline Cirurgia mamária prévia & & 0,005 \\
\hline Não & $7,1(6,9 ; 7,3)$ & \\
\hline Sim & $6,4(6,0 ; 6,8)$ & \\
\hline IG da criança ao nascimento & & 0,011 \\
\hline$<37$ semanas & $6,0(5,3 ; 6,8)$ & \\
\hline$\geq 37$ semanas & $7,0(6,8 ; 7,2)$ & \\
\hline Peso ao nascer & & 0,006 \\
\hline$<2.500$ gramas & $5,6(4,8 ; 6,6)$ & \\
\hline$\geq 2.500$ gramas & $7,0(6,8 ; 7,2)$ & \\
\hline Apgar de $1^{\circ}$ minuto & & 0,270 \\
\hline$<7$ & $6,5(5,7 ; 7,4)$ & \\
\hline$\geq 7$ & $7,0(6,8 ; 7,2)$ & \\
\hline Horas de vida da criança & & $<0,001$ \\
\hline$<24$ horas & $6,3(6,0 ; 6,6)$ & \\
\hline 24 a 48 horas & $7,1(6,8 ; 7,4)$ & \\
\hline$>48$ horas & $7,4(7,1 ; 7,8)$ & \\
\hline
\end{tabular}

Modelo linear generalizado com distribuição de probabilidade gama e função de ligação log. IG - Idade gestacional

Tabela 5. Valores médios e Intervalo de Confiança 95\% (IC 95\%) para o escore total da escala LATCH segundo as variáveis sociodemográficas, clínicas e obstétricas e 0 tempo de vida da criança

\begin{tabular}{|c|c|c|c|}
\hline \multirow{2}{*}{ Variáveis } & \multicolumn{3}{|c|}{ Horas de vida da criança } \\
\hline & $<24$ horas & 24 a 48 horas & $>48$ horas \\
\hline \multicolumn{4}{|l|}{ Idade materna } \\
\hline$<40$ anos & $6,3(6,0 ; 6,6)$ & $7,1(6,8 ; 7,4)$ & $7,4(7,0 ; 7,8)$ \\
\hline$\geq 40$ anos & - & $7,3(6,0 ; 8,9)$ & $7,7(6,7 ; 8,8)$ \\
\hline$p$-value & - & 0,756 & 0,629 \\
\hline \multicolumn{4}{|l|}{ Paridade } \\
\hline Primípara & $6,3(5,9 ; 6,8)$ & $7,4(7,0 ; 7,9)$ & $7,5(7,0 ; 8,1)$ \\
\hline Multípara & $6,3(5,8 ; 6,7)$ & $6,8(6,5 ; 7,2)$ & $7,4(6,9 ; 7,9)$ \\
\hline$p$-value & 0,869 & 0,032 & 0,716 \\
\hline \multicolumn{4}{|l|}{ Tipo de parto } \\
\hline Vaginal & $6,6(6,0 ; 7,3)$ & $7,5(6,9 ; 8,0)$ & $7,5(6,7 ; 8,3)$ \\
\hline Cesárea & $6,1(5,8 ; 6,5)$ & $7,0(6,6 ; 7,3)$ & $7,4(7,0 ; 7,9)$ \\
\hline$p$-value & 0,194 & 0,125 & 0,924 \\
\hline \multicolumn{4}{|c|}{ Cirurgia mamária prévia } \\
\hline Não & $6,4(6,0 ; 6,8)$ & $7,2(6,9 ; 7,5)$ & $7,5(7,1 ; 7,9)$ \\
\hline Sim & $5,9(5,3 ; 6,5)$ & $6,5(6,0 ; 7,2)$ & $7,0(6,2 ; 7,9)$ \\
\hline$p$-value & 0,170 & 0,048 & 0,317 \\
\hline \multicolumn{4}{|c|}{ IG da criança ao nascer } \\
\hline$<37$ semanas & - & $6,0(5,2 ; 6,9)$ & $6,0(5,0 ; 7,3)$ \\
\hline$\geq 37$ semanas & $6,3(6,0 ; 6,6)$ & $7,2(6,9 ; 7,5)$ & $7,5(7,1 ; 7,9)$ \\
\hline$p$-value & - & 0,006 & 0,014 \\
\hline
\end{tabular}

Modelo linear generalizado com distribuição de probabilidade gama e função de ligação log. IG - Idade gestacional 


\section{Discussão}

A maternidade em que foi realizado o estudo representa uma população de nível socioeconômico mais alto que destoa da realidade do país, mas não anula a magnitude dos achados científicos encontrados, por se tratar do primeiro estudo que investiga os parâmetros de avaliação da escala LATCH no Brasil. Um número amostral maior e com diferentes populaçóes poderia ampliar estes resultados (Apêndice 1).

A LATCH representa uma ferramenta simples para avaliar e monitorar a técnica de amamentação de maneira mais objetiva pelo enfermeiro, ${ }^{(2,13)}$ de forma a contribuir com a rotina de avaliação e planejamento da assistência de enfermagem, na priorização do cuidado da mulher que amamenta na maternidade.

As dificuldades da técnica da amamentação pela escala LATCH foram: pega inadequada, deglutição não audível, com conforto e posicionamento inadequado. $\mathrm{O}$ tipo de mamilo não é citado em relação à dificuldade de amamentação. Existe também uma considerável relação entre o tempo de vida e o escore total. Quanto maior a idade da criança, maior o escore total LATCH. A nota de corte identificada foi 6 , e a tendência é quanto maior as horas de vida, maior o escore, com base nas variáveis que interferem: presença de cirurgia mamária, criança com idade gestacional $<37$ semanas e peso ao nascer $<2.500$ gramas.

Os parâmetros de pega e conforto se comportaram de forma relacionada, no qual a pega apresenta mudança significativa no escore entre $<24$ horas e $>48$ horas. Este achado corrobora com a literatura, na qual observa-se melhora da pega com o passar dos dias de prática da criança, enquanto o conforto é maior no período inicial em que os mamilos estão íntegros e, o aumento da exposiçáo persistente da pega incorreta, reduz o escore individual deste item. ${ }^{(14,15)}$ Estudo mostra que a queixa de dor e lesão mamilar nos primeiros dias pós-parto é muito frequente, cuja média de dor encontrada foi de $6.2 .{ }^{(14)} \mathrm{A}$ taxa desta queixa é crescente aos longo dos dias, e seu ápice é alcançado no quarto dia pós-parto, ${ }^{(15)}$ o que reforça a importância da identificação precoce da dor e/ou lesão mamilar para evitar complicaçóes. ${ }^{(16)} \mathrm{O}$ manejo da lactação no primeiro dia pós-parto é eficaz para a prevenção da dor nos mamilos, e seu diagnóstico e tratamento efetivo são cruciais para evitar o desmame. ${ }^{(17,18)}$

Por outro lado, nos parâmetros de posicionamento e deglutição, as diferenças encontradas demonstraram melhoria dos itens de forma crescente. Estas diferenças foram identificadas no escore total LATCH quando comparadas crianças entre $<24$, 24-28 e >48 horas de vida. Estes achados vão de encontro com a literatura em que há melhora da prática do posicionamento na mamada, conforme o tempo de experiência da dupla máe-filho, ${ }^{(2,16,17)}$ assim como decorrente da apojadura a partir de 48 horas pós-parto, o que naturalmente aumenta o volume de leite ingerido pela criança e facilita a ausculta da deglutição pelo profissional. ${ }^{(19,20)}$ Vale destacar que a deglutição pode ser avaliada com o uso de um estetoscópio, para facilitar a ausculta nas primeiras mamadas em que o volume de leite é menor. ${ }^{(2)}$

No que se refere ao tipo de mamilo, apesar do presente estudo não ter identificado como uma dificuldade pelo escore LATCH, sabe-se que àqueles classificados como malformados (invertidos e subdesenvolvidos) estão relacionados à maiores problemas para amamentar, ao passo que os mamilos protrusos podem ser facilitadores. ${ }^{(17,20,21)} \mathrm{O}$ resultado encontrado provavelmente justifica-se pela maioria (98\%) das mulheres estudadas apresentarem mamilos protrusos.

Além da avaliação por item da escala, a identificaçáo do escore total pode ajudar no monitoramento diário da dupla quanto à técnica da mamada. O presente estudo identificou que há aumento progressivo do escore total ao longo dos dias de vida da criança, resultado converge com a literatura que mostra uma melhora da técnica ao longo dos dias de vida. ${ }^{(22)}$ As crianças com $>48$ horas apresentaram escore total superior a $5(\geq 6, \geq 7 \mathrm{e}$ $\geq 8$ ) quando comparado àquelas com $<24$ horas. Este achado contribui para um direcionamento na definição da nota de corte dos escores apresentados pela dupla mãe-filho, em uma avaliação de mamada, no qual um escore total mínimo de 6 pode ser classificado como satisfatório. Este resultado foi congruente com Shah et al., ${ }^{(22)}$ que demonstraram 
que o escore LATCH $>6$ na alta aumenta cinco vezes a chance de ocorrer aleitamento materno exclusivo na sexta semana pós-parto. Assim como Sowjanya et al., ${ }^{(3)}$ que identificaram uma sensibilidade de $93 \%$ e especificidade de $66 \%$ para o escore $\geq 6$ ao nascer, enquanto para a alta (48 horas pós-parto) o LATCH $\geq 8$ foi de $94 \%$ e $82 \%$ respectivamente, aumentando em nove vezes a chance de aleitamento materno nas $6^{\text {a }}$ semana pós-parto. Por outro lado, Sowjanya et al., ${ }^{(3)}$ sugerem que a escala LATCH seja interpretada considerando a classificação: Ruim (0-3), Moderada (4-7) e Boa (8-10), no entanto, apesar desta classificação direcionar objetivamente o enfermeiro, o estudo náo detalha como as categorias foram determinadas e limita seu uso na prática. Jensen et al., ${ }^{(2)} \mathrm{a}$ idealizadora da escala LATCH, enfatiza a importância da correção de todos os itens de forma individual, quando necessário, visto a importância da correçáo das dificuldades encontradas de forma pontual e global. Sendo assim, é possível afirmar que o escore total $\geq 6$ é um bom ponto de corte para identificar as necessidades de maior apoio da equipe no hospital, sem perder o olhar individual de cada item da escala. Novos estudos podem explorar os pontos de corte e relacionar com as condutas dos profissionais que apontam para a amamentação, proporcionando assim um melhor direcionamento da assistência sistematizada na maternidade pelo enfermeiro.

Quanto à relação entre o escore total da LATCH e as características sociodemográficas, foi observado que as mulheres que possuíam cirurgia mamária, crianças nascidas com idade gestacional $<37$ semanas e peso ao nascer $<2.500$ gramas apresentaram menor escore. Este resultado demonstra a necessidade de priorizar o atendimento desta dupla durante a internaçáo, confirmado pela literatura, em que as crianças prematuras e pequenas para a idade gestacional apresentam mais dificuldades para amamentar, tais como sonolência, manutenção da pega, sucção e frequência da mamada. ${ }^{(23,24)}$ No que se refere à cirurgia mamária, apesar da literatura não ser consistente para a confirmaçáo de quais questóes relacionadas aos itens da escala LATCH com a cirurgia, estudos demonstram que há maior risco da criança não estar em aleitamento materno exclusivo. ${ }^{25,26)}$

A ferramenta LATCH demonstrou ser útil para o planejamento da assistência do enfermeiro para as mulheres que amamentam. Os baixos escores do LATCH indicam a necessidade de intervenção ativa, no auxílio da identificação e direcionamento de mães em risco de desmame precoce. ${ }^{(27)}$

\section{Conclusão}

O uso do LATCH foi útil para verificar a relação entre dificuldades das mulheres relacionadas à técnica e características sociodemográficas, obstétricas e neonatais durante a hospitalização. A avaliação dos itens pega, deglutição, conforto e posicionamento variam de acordo com as horas de vida, e deve ser considerada individualmente, além do parâmetro do escore total, com objetivo de direcionar a equipe de enfermagem no planejamento do cuidado diário na maternidade. Para os recém-nascidos com $<24$ horas, é preciso atenção quanto ao posicionamento correto e orientação quanto à melhor prática da amamentação. Os grupos de mulheres que possuem cirurgia de mama, crianças nascidas com $<37$ semanas e com peso $<2.500$ gramas podem necessitar maior atenção do enfermeiro.

\section{Agradecimentos}

As autoras agradecem todo o suporte oferecido pelo Hospital Israelita Albert Einstein e, em especial, para a Enfermeira Simone Brandi pelo apoio na realização da pesquisa.

\section{Colaborações}

Griffin CMC, Amorim MHC, Almeida FA, Marcacine KO, Goldman RE e Coca KP contribuíram com a concepção do estudo, análise e interpretação dos dados, redação do artigo, revisão crítica do conteúdo intelectual e aprovação da versão final a ser publicada. 


\section{Referências}

1. Sartorio BT, Coca KP, Marcacine KO, Abuchaim ÉS, Abrão AC. Breastfeeding assessment instruments and their use in clinical practice. Rev Gaucha Enferm. 2017;38(1):e64675.

2. Jensen D, Wallace S, Kelsay P. LATCH: a breastfeeding charting system and documentation tool. J Obstet Gynecol Neonatal Nurs. 1994;23(1):27-32.

3. Sowjanya SV, Venugopalan L. LATCH score as a predictor of exclusive breastfeeding at 6 weeks postpartum: a prospective cohort study. Breastfeed Med. 2018;13(6):444-9.

4. Kumar SP, Mooney R, Wieser LJ, Havstad S. The LATCH scoring system and prediction of breastfeeding duration. J Hum Lact. 2006;22(4):391-7.

5. Riordan J, Bibb D, Miller M, Rawlins T. Predicting breastfeeding duration using the LATCH breastfeeding assessment tool. J Hum Lact. 2001;17(1):20-3.

6. Altuntas N, Turkyilmaz C, Yildiz H, Kulali F, Hirfanoglu I, Onal E, et al. Validity and reliability of the infant breastfeeding assessment tool, the mother baby assessment tool, and the LATCH scoring system. Breastfeed Med. 2014;9(4):191-5.

7. Freitas MG, Werneck AL, Borim BC. Exclusive breastfeeding: adhesion and difficulties. J Nurs UFPE Online. 2018;12(9):2301-7.

8. Tornese G, Ronfani L, Pavan C, Demarini S, Monasta L, Davanzo R. Does the LATCH score assessed in the first 24 hours after delivery predict non-exclusive breastfeeding at hospital discharge? Breastfeed Med. 2012;7(6):423-30.

9. Dolgun G, İnal S, Erdim L, Korkut S. Reliability and validity of the Bristol Breastfeeding Assessment Tool in the Turkish population. Midwifery. 2018:57:47-53.

10. Conceição CM, Coca KP, Alves MR, Almeida FA Validation of the LATCH breastfeeding assessment instrument for the Portuguese language. Acta Paul Enferm. 2017;30(2):210-6.

11. Faraway JJ. Extending the linear model with R: generalized linear, mixed effects and nonparametric regression models. United Kingdom: Chapman \& Hall; 2006

12. Shoukri MM, Asyali MH, Donner A. Sample size requirements for the design of reliability study: review and new results. Stat Methods Med Res. 2004;13 (4):251-71.

13. Lau Y, Htun TP, Lim PI, Ho-Lim S, Klainin-Yobas P. Psychometric evaluation of 5 and 4 item versions of the LATCH breastfeeding assessment tool during the initial postpartum period among a multiethnic population. Plos One. 2016;11(5):e0154331.

14. Coca KP, Amir LH, Alves MD, Barbieri M, Marcacine KO, de Vilhena Abrão AC. Measurement tools and intensity of nipple pain among women with or without damaged nipples: a quantitative systematic review. J Adv Nurs. 2019;75(6):1162-72.

15. Nakamura M, Asaka Y, Ogawara T, Yorozu Y. Nipple skin trauma in breastfeeding women during postpartum week one. Breastfeed Med. 2018;7(13):479-84

16. Berens P, Eglash A, Malloy M, Steube AM. ABM Clinical Protocol \#26: Persistent Pain with Breastfeeding. Breastfeed Med. 2016;11(2):4653. Review.

17. Miss AV, Shobiri F, Masoumi SZ, Mohammadi Y. Effect of Teaching Breastfeeding Technique on Breastfeeding Position in Primiparous Women. J Educ Community Health. 2019;6(1):11-15.

18. Dias JS, Vieira TO, Vieira GO. Factors associated to nipple trauma in lactation period: a systematic review. Rev Bras Saude Mater Infant. 2017;17(1):27-42. Review.
19. Huang L, Xu S, Chen X, Li Q, Lin L, Zhang Y, et al. Delayed lactogenesis is associated with suboptimal breastfeeding practices: a prospective cohort study. J Nutr. 2020;150(4):894-900

20. Carreiro JA, Francisco AA, Abrão AC, Marcacine KO, Abuchaim ES, Coca KP. Breastfeeding diffi culties: analysis of a service specialized in breastfeeding. Acta Paul Enferm. 2018;31(4),430-8.

21. Kent JC, Ashton E, Hardwick CM, Rowan MK, Chia ES, Fairclough KA, et al. Nipple pain in breastfeeding mothers: incidence, causes and treatments. Int J Environ Res Public Health. 2015;12(10):12247-63.

22. Shah MH, Roshan R, Parikh T, Sathe S, Vaidya U, Pandit A. LATCH score at discharge: a predictor of weight gain and exclusive breastfeeding at 6 weeks in term healthy babies. J Pediatr Gastroenterol Nutr. 2021;72(2):e48-e52.

23. Lau C. Breastfeeding challenges and the preterm mother-infant dyad: a conceptual model. Breastfeed Med. 2018;13(1):8-17. Review.

24. Cartwright J, Atz T, Newman S, Mueller M, Demirci JR. Integrative review of interventions to promote breastfeeding in the late preterm infant. J Obstet Gynecol Neonatal Nurs. 2017;46(3):347-56. Review.

25. Kraut RY, Brown E, Korownyk C, Katz LS, Vandermeer B, Babenko 0, et al. The impact of breast reduction surgery on breastfeeding: Systematic review of observational studies. PLoS One. 2017;12(10):e0186591. Review.

26. Marcacine KO, Abuchaim ES, Coca KP, Abrão AC. Factors associated to breast implants and breastfeeding. Rev Esc Enferm USP. 2018;52:e03363.

27. Karthika S, Mathivanan M, Maheswari K, Hiremath PB, Jesintha DM. A study on role of LATCH scoring in duration of exclusive breastfeeding in a rural tertiary care hospital, Puducherry: a prospective study. Int J Contemp Pediatrics. 2020;7(1):198-202.

Apêndice 1. Características sociodemográficas, clínicas e obstétricas por tempo de vida da criança no momento da coleta

\begin{tabular}{|c|c|c|c|c|}
\hline \multirow[b]{2}{*}{ Variáveis } & \multicolumn{3}{|c|}{ Horas de vida da criança } & \multirow[b]{2}{*}{$\begin{array}{c}\text { Total } \\
(n=162)\end{array}$} \\
\hline & $\begin{array}{c}<24 \text { horas } \\
(n=43)\end{array}$ & $\begin{array}{c}24 \text { a } 48 \\
\text { horas } \\
(n=74)\end{array}$ & $\begin{array}{c}>48 \text { horas } \\
(n=45)\end{array}$ & \\
\hline \multicolumn{5}{|l|}{ Idade materna } \\
\hline$<40$ anos & 43 & 71 & 39 & 153 \\
\hline$\geq 40$ anos & 0 & 3 & 6 & 9 \\
\hline \multicolumn{5}{|l|}{ Paridade } \\
\hline Primípara & 23 & 34 & 20 & 77 \\
\hline Multípara & 20 & 40 & 25 & 85 \\
\hline \multicolumn{5}{|l|}{ Tipo de parto } \\
\hline Vaginal & 13 & 22 & 11 & 46 \\
\hline Cesárea & 30 & 52 & 34 & 116 \\
\hline \multicolumn{5}{|c|}{ Cirurgia mamária prévia } \\
\hline Não & 32 & 61 & 38 & 131 \\
\hline $\operatorname{Sim}$ & 11 & 13 & 7 & 31 \\
\hline \multicolumn{5}{|c|}{ IG da criança ao nascer } \\
\hline$<37$ semanas & 0 & 6 & 3 & 9 \\
\hline$\geq 37$ semanas & 43 & 68 & 42 & 153 \\
\hline \multicolumn{5}{|l|}{ Peso ao nascer } \\
\hline$<2.500$ gramas & 1 & 1 & 3 & 5 \\
\hline$\geq 2.500$ gramas & 42 & 73 & 42 & 157 \\
\hline \multicolumn{5}{|l|}{ Apgar de $1^{\circ}$ minuto } \\
\hline$<7$ & 1 & 5 & 2 & 8 \\
\hline$\geq 7$ & 42 & 69 & 43 & 154 \\
\hline
\end{tabular}

